Revista Universo Contábil, ISSN 1809-3337

Blumenau, v. 14, n. 4, p. 50-67, out./dez., 2018

doi:10.4270/ruc. 2018427

Disponível em www.furb.br/universocontabil

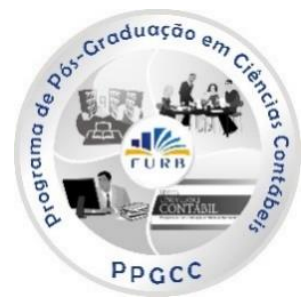

\title{
ANÁLISE DE MÉTRICAS PARA A INTENSIDADE DE ATIVOS NO COMPORTAMENTO ASSIMÉTRICO DOS CUSTOS 1
}

\section{ANALYSIS OF METRICS FOR THE INTENSITY OF ASSETS ON COST STICKINESS \\ ANÁLISIS DE MÉTRICAS PARA LA INTENSIDAD DE ACTIVOS EN LOS COSTOS PEGAJOSOS}

\begin{abstract}
Altair Borgert
Doutor em Engenharia de Produção pela Universidade Federal de Santa Catarina Professor do PPG em Contabilidade na Universidade Federal de Santa Catarina Endereço: UFSC, Centro Socioeconômico, Bloco C, Sala 201 -Campus Universitário - Trindade CEP: 88010-970 - Florianópolis - SC - Brasil E-mail: altair@borgert.com.br Telefone: (48) 3721-6671

Thayse Moraes Elias Mestranda do PPG em Contabilidade na Universidade Federal de Santa Catarina Endereço: UFSC, Centro Socioeconômico, Bloco C, Sala 201 -Campus Universitário - Trindade CEP: 88010-970 - Florianópolis - SC - Brasil E-mail: thaysemooraes@hotmail.com Telefone: 48 3721-6671

Luiza Santangelo Reis Mestre em Contabilidade pela Universidade Federal de Santa Catarina Doutoranda do PPG em Contabilidade na Universidade Federal de Santa Catarina Endereço: UFSC, Centro Socioeconômico, Bloco C, Sala 201 -Campus Universitário - Trindade CEP: 88010-970 - Florianópolis - SC - Brasil E-mail: thaysemooraes@hotmail.com Telefone: 48 3721-6671
\end{abstract}

\section{RESUMO}

O objetivo deste estudo consiste em analisar a representatividade das diferentes métricas para a intensidade de ativos no comportamento assimétrico dos custos, com destaque para: (i) razão do Ativo Total pela Receita Líquida de Vendas; (ii) razão do Imobilizado pela Receita Líquida de Vendas; e (iii) razão do Imobilizado pelo Ativo Total. Por meio de um levantamento de informações das empresas listadas na BM\&FBOVESPA, durante um período de 21 anos, empregou-se o modelo de regressão para dados em painel para identificar se as variáveis

\footnotetext{
${ }^{1}$ Artigo recebido em 06/12/2017. Revisado por pares em 12/03/2019. Reformulado em 21/03/2019. Recomendado para publicação em 29/03/2019 por Franciele Beck. Publicado em 13/05/2019. Organização responsável pelo periódico: FURB.
} 
inseridas são significativas e o quanto influenciam na assimetria dos custos. Os resultados indicam a proxy razão do Ativo Total pela Receita Líquida de Vendas como a mais representativa para a análise da influência da intensidade de ativos, tanto no comportamento do Custo dos Produtos Vendidos como das Despesas com Vendas e Administrativas para as empresas estudadas, o que pode servir de suporte para as pesquisas em que a proxy para intensidade de ativos é aplicada. Assim, por meio dessa métrica conclui-se que, quanto maior a intensidade de ativos, maior a assimetria dos custos e das despesas no sentido Sticky Costs.

Palavras-chave: Assimetria dos custos; Sticky costs; Intensidade de ativos.

\section{ABSTRACT}

The objective of this study is to analyze the representativeness of the different metrics that measure the asset intensity on asymmetric cost behavior. The measure we analyze are: (i) ratio of Total Assets to Net Sales Revenue; (ii) ratio of Property, Plant and Equipment to Net Sales Revenue; and (iii) ratio of Property, Plant and Equipment to Total Assets. We analyze the data from a survey of the companies listed on BM\&FBOVESPA by panel data regression, during a period of 21 years, to identify if the inserted variables are significant and how much it influence the expenses asymmetry. The results show that the ratio of Total Asset to Net Sales Revenue is a more representative measure to analyze the influence of the asset intensity on the behavior of Cost of Goods Sold and Selling, General and Administrative Expenses, which can be used as support for searches in which the proxy for the asset intensity is applied. Thus, through this proxy we conclude the greater the asset intensity, the greater the asymmetry of costs and expenses in the direction of Sticky Costs.

Keywords: Cost stickiness; Sticky Costs; Asset intensity.

\section{RESUMEN}

El objetivo de este estudio consiste en analizar la representatividad de las diferentes métricas para la intensidad de activos en el comportamiento asimétrico de los costos, con destaque para: (i) proporción del Activo Total por la Receita Líquida de Ventas; (ii) proporción del Inmovilizado por la Receita Líquida de Ventas; y (iii) proporción del Inmovilizado por el Activo Total. Por medio de un relevamiento de informaciones de las empresas listadas en la BM\&FBOVESPA, durante un período de 21 años, se empleó el modelo de regresión para datos en panel, para identificar si las variables insertadas son significativas y cuán influyen en la asimetría de los gastos. Los resultados indican el proporción del Activo Total por la Receta Líquida de Ventas como el más representativa para el análisis de la influencia de la intensidad de activos tanto en el comportamiento del Costo de los Productos Vendidos como de los Gastos con Ventas y Administrativas para las empresas estudiadas, lo que puede servir de soporte para las búsquedas en las que se aplica el proxy para la intensidad de activos. Así, por medio de esta métrica se concluye que, cuanto mayor la intensidad de activos, mayor es el costos pegajosos y de los gastos en el sentido Sticky Costs.

Palabras clave: Costos pegajosos; Sticky costs; Intensidad de activos.

\section{INTRODUÇÃO}

Tradicionalmente, a abordagem do comportamento dos custos presumia uma variação simétrica dos custos em relação a mudanças no volume de atividades, independentemente da sua direção, para mais ou para menos. Nessa perspectiva, de acordo com Banker e Byzalov (2014), basicamente, os custos são classificados como variáveis - na medida em que se alteram de acordo com a variação no volume de atividades - e fixos - na medida em que não se alteram na mesma proporção. 
Em outra perspectiva, Anderson, Banker e Janakiraman (2003) publicaram evidências de que a relação entre os custos e o volume de atividades é assimétrica ao identificar que quando a receita aumenta $1 \%$, as despesas com vendas, gerais e administrativas aumentam $0,55 \%$, porém, quando a receita diminui $1 \%$, as despesas diminuem somente $0,35 \%$. Esse fenômeno foi denominado pelos referidos autores de sticky costs, cuja magnitude do aumento dos custos em resposta ao aumento da receita é maior do que a magnitude da redução dos custos relacionada à redução da receita, para o mesmo percentual de variação da receita.

Além desse comportamento no sentido sticky costs, outros estudos, como o de Weiss (2010) por exemplo, detectaram que o fenômeno pode ocorrer no sentido inverso e o denominaram de anti-sticky costs. Ou seja, a magnitude do aumento dos custos relacionada ao aumento da receita pode ser menor do que a magnitude da redução dos custos associada à redução da receita. Ainda, nessa direção, Abu-Serdaneh (2014) menciona que o grau de assimetria pode variar para diferentes contas de custos, empresas e países, o que resulta num comportamento sticky, anti-sticky ou simétrico.

Contudo, a evidenciação da assimetria no comportamento dos custos precedeu a expansão das análises em diversos países, nos quais pesquisadores detectaram a sua existência em diferentes setores e segmentos empresariais. Dentre outros, Subramaniam e Weidenmier (2003) argumentam que a assimetria dos custos pode variar entre empresas e setores devido, sobretudo, às peculiaridades de cada negócio. Na sequência, conforme Silva et al. (2019), os estudos se voltaram para a análise dos fatores que interferem no comportamento assimétrico dos custos, principalmente quando da ocorrência de mudanças nos níveis de atividades organizacionais.

Um dos fatores mais discutidos na literatura diz respeito aos custos de ajustamento relacionados às adaptações de recursos quando das alterações na demanda. Anderson, Banker e Janakiraman (2003) explicam que quando há queda no volume os gestores optam por manter os recursos comprometidos e arcam com os custos da capacidade não utilizada, ou reduzem os recursos e incorrem em custos de ajustamento inerentes a redução e substituição dos recursos comprometidos, caso a demanda seja recuperada. Neste sentido, conforme os autores, a assimetria dos custos pode ser mais acentuada nos casos em que os custos de adaptação dos recursos comprometidos sejam elevados, pois os gestores optam por manter recursos não utilizados.

Anderson, Banker e Janakiraman (2003) argumentam, ainda, que os custos de ajustamento provavelmente são maiores quando as empresas contam com mais ativos próprios e mais empregados. A redução dos recursos adquiridos de terceiros, quando há redução da demanda, é relativamente fácil, a menos que existam contratos de longo prazo. No entanto, a alienação dos ativos próprios é dispendiosa, devido aos custos inerentes à venda e à perda dos investimentos relacionados com a instalação e personalização dos seus ativos. Essas hipóteses foram confirmadas pelos referidos autores quando encontraram coeficientes significativos e negativos que indicaram que os custos são mais inflexíveis em empresas que exigem, relativamente, mais ativos ou mais empregados para apoiar suas vendas.

Além de Anderson, Banker e Janakiraman (2003), outros autores encontraram resultados similares, no sentido de que a intensidade de ativos aumenta o grau de assimetria dos custos, como Subramaniam e Weidenmier (2003), Calleja, Steliaros e Thomas (2006), He, Teruya e Shimizu (2010), Porporato e Werbin (2012), Banker, Fang e Mehta (2013), AbuSerdaneh (2014), Dalla Via e Perego (2014), Jalilian e Elyssai (2014) e Richartz (2016).

Todavia, as medidas utilizadas para representar a intensidade de ativos nem sempre são similares, uma vez que alguns autores empregaram a razão do ativo total pela receita de vendas, como Anderson, Banker e Janakiraman (2003), He, Teruya e Shimizu (2010), Dalla Via e Perego (2014), enquanto outros utilizaram a razão do ativo imobilizado pela receita de vendas, como Subramaniam e Weidenmier (2003) e Jalilian e Elyssai (2014). Também, a razão do ativo 
imobilizado pelo ativo total é empregada por alguns autores como Banker, Fang e Mehta (2013) e Richartz (2016). Além dessas, pode-se observar na literatura a utilização de outras métricas menos recorrentes como giro do ativo imobilizado e giro do estoque, razão do ativo total pelo lucro operacional e gastos com manutenção de ativos.

Essa ausência de consenso pode ser observada na literatura pertinente à conceituação de ativos. Assim, conforme Hendriksen e Van Breda (2009), a preocupação com conceitos explícitos de ativos e passivos, e uma verificação de suas características de natureza básica, se justificam com o propósito de contribuir com a teoria da contabilidade, por meio de diretrizes adequadas, para o desenvolvimento do pensamento contábil e dos próprios princípios de contabilidade.

O Pronunciamento Conceitual Básico (CPC, 2011) conceitua o ativo como um recurso controlado pela entidade como resultado de eventos passados e do qual se espera que fluam futuros benefícios econômicos para a entidade. Por outro lado, Canning (1929) conceitua ativo como qualquer serviço futuro, em termos monetários ou controlável em moeda, cujos direitos pertencem legal ou justamente à alguma pessoa ou a um conjunto de pessoas, e o serviço é um ativo somente para quem o usufruir. Pode-se perceber que as duas conceituações apresentam diferenças relacionadas à delimitação de ativo.

As diversas métricas para a intensidade de ativos possuem diferenças em termos informacionais e, consequentemente, diferentes potenciais de informação devido, sobretudo, ao fato de serem aplicadas em diferentes contextos regionais. Pesquisadores devem determinar qual a métrica que pretendem aplicar nos estudos com base nos conceitos envolvidos e no direcionamento das suas discussões. No entanto, os aspectos conceituais inerentes às métricas são pouco abordados nas pesquisas, bem como não há padronização para a proxy de intensidade de ativos.

Nessa linha de discussão, a seguinte pergunta norteia a presente pesquisa: qual métrica para a intensidade de ativos é a mais representativa para a explicação da assimetria dos custos e das despesas das empresas listadas na BM\&FBOVESPA?

Portanto, o objetivo do presente estudo consiste em verificar qual das métricas comumente utilizadas para a intensidade de ativos no comportamento assimétrico dos Custos dos Produtos Vendidos e das Despesas com Vendas e Administrativas, das empresas listadas na BM\&FBOVESPA, se mostra mais representativa. Por meio da significância e do coeficiente pretende-se contribuir para a lacuna existente, sobre qual é a métrica mais representativa para a intensidade de ativos, uma vez que os estudos em geral focam na análise da intensidade de ativos na assimetria dos custos, bem como utilizam diferentes métricas para análise sem um questionamento sobre a sua relevância.

Em termos teóricos, a justificativa deste estudo se fundamenta na necessidade de desenvolvimento da literatura sobre comportamento dos custos, especialmente quanto à abordagem da assimetria dos custos, ao considerar as particularidades de cada fator explicativo para o fornecimento de subsídios que possibilitam melhoria no gerenciamento dos custos das empresas. Em termos práticos, a pesquisa se justifica pelo melhor entendimento dos custos e do poder preditivo dos lucros, uma vez que, conforme o estudo de Weiss (2010), as empresas que apresentam comportamento mais assimétrico têm previsões menos precisas comparativamente às empresas que apresentam menos assimetria.

Além disso, ao considerar a discussão acerca das métricas para a intensidade de ativos e ao fornecer indícios de qual a mais representativa, a pesquisa contribui para o avanço das pesquisas relacionadas ao comportamento dos custos e demais estudos que empregam alguma proxy para a intensidade de ativos em âmbito nacional e internacional. Ainda, destaca-se que no Brasil as pesquisas empregam a utilização de proxy para intensidade de ativos, mas não foram encontrados estudos que discutem a representatividade de métricas para os fatores inerentes ao comportamento dos custos estudados. 
A delimitação deste estudo se dá em função das empresas e da variável observada, bem como do tempo. Devido à disponibilidade das informações, as empresas abrangidas na análise são as listadas na BM\&FBOVESPA e a variável analisada é a intensidade de ativos, ao considerar o objetivo deste estudo. Quanto à delimitação temporal, definiu-se uma amostra de 21 anos após a adoção do plano real em que o Brasil viveu um período de maior estabilidade econômica, o qual previne que as observações não sejam enviesadas por medidas gerenciais adotadas em decorrência das altas taxas inflacionárias.

\section{FUNDAMENTAÇÃO TEÓRICA}

O estudo de Anderson, Banker e Janakiraman (2003), além de impulsionar a verificação da assimetria dos custos, motivou a análise da intensidade de ativos ao constatar a influência dessa variável na assimetria das despesas. Nessa direção, Banker, Byzalovy e Plehn-Dujowichz (2010) apresentaram evidências empíricas de estrutura de custos rígidas em países como a Austrália, Alemanha, França, UK, Hong Kong, Japão, Suécia, Tailândia e África do Sul. Uma das hipóteses testadas no estudo sugere que o grau de assimetria dos custos aumenta com os custos de ajustamento, que foram medidos por meio da intensidade de ativos e intensidade de funcionários. Em relação a isso, Banker, Byzalovy e Plehn-Dujowichz (2010) encontraram que maior intensidade de ativos está associada ao maior grau de assimetria dos custos para a maior parte dos países na amostra.

Ao investigar os determinantes dos sticky costs em empresas Tailandesas, Pichetkun (2012) encontrou resultados que indicam que os custos de ajustamento, por meio da intensidade de ativos, são positivamente associados com o grau de assimetria dos custos. Da mesma forma, Banker e Byzalov (2014) verificaram que o grau de assimetria dos custos aumenta com o aumento nos custos de ajustamento de recursos. Os autores utilizaram como proxy para custos de ajustamento de recursos a intensidade de ativos e a intensidade de empregados, uma vez que os custos de ajustamento não são diretamente observáveis, cujos resultados indicaram que a assimetria no sentido sticky costs é mais elevada para empresas intensivas no uso de ativos e empregados. Por fim, Banker e Byzalov (2014) concluíram que o grau de assimetria dos custos em cada país é heterogêneo entre as empresas, bem como varia sistematicamente com os custos de ajustamento.

Já, Venieris, Naoum e Vlismas (2015) pesquisaram a influência dos níveis de ativos intangíveis na folga dos recursos, cujos resultados indicaram que, para empresas com elevado capital, as despesas com vendas, gerais e administrativas apresentam comportamento sticky costs, e para empresas com baixo capital, as despesas apresentam comportamento anti-sticky. Além dos ativos intangíveis como medida para a intensidade do capital, a intensidade de ativos foi inserida nas análises como uma variável para custos de ajustamento, no referido estudo.

Paralelamente, ao analisar o conservadorismo e o comportamento dos custos, Banker et al. (2016) encontraram resultados relacionados aos custos de ajustamento que apontam, também, que a assimetria aumenta com a intensidade de ativos e com a intensidade de empregados.

Subramaniam e Weidenmier (2003) analisaram os custos dos produtos vendidos e as despesas com vendas, gerais e administrativas de empresas dos setores de fabricação, merchandising, empresas de serviços e serviços financeiros e verificaram diferenças entre tais empresas com relação ao comportamento assimétrico dos custos, com base em alguns determinantes que são suscetíveis à variabilidade entre empresas, dos quais um deles é a intensidade de ativos fixos das empresas. Os resultados indicam que as empresas de fabricação são as que apresentam comportamento mais assimétrico, devido aos altos níveis de ativos fixos e inventário. Por outro lado, empresas de merchandising e de serviços apresentam baixos níveis de assimetria devido aos baixos níveis de ativos fixos e uso de ajuda temporária, que permite respostas rápidas às mudanças significativas na receita. 
Além disso, Subramaniam e Weidenmier (2003) argumentam que, enquanto grandes aumentos de atividade resultam em aumentos imediatos de custos, grandes reduções podem não resultar em reduções imediatas de custos, mesmo quando existe capacidade ociosa significativa, porque as empresas podem optar por não reduzir os ativos fixos bem como outros custos no curto prazo. Assim, os resultados apontam que para dois períodos consecutivos de queda da receita a assimetria é menor, o que significa que no curto prazo pode ser difícil reduzir os custos de capacidade relacionados ao ativo imobilizado.

Em contexto mais amplo, Calleja, Steliaros e Thomas (2006) investigaram o comportamento dos custos de empresas dos Estados Unidos, Reino Unido, França e Alemanha e encontraram que os custos operacionais são assimétricos. Como resultado, os autores destacam evidências que sugerem a existência do efeito das características específicas das empresas e que os dados apoiam que a assimetria dos custos está associada a empresas com alta intensidade de ativos e, em menor escala, a alta intensidade de empregados. Uma justificativa levantada por Calleja, Steliaros e Thomas (2006), para a relação positiva entre a intensidade de ativos e o grau de assimetria, diz respeito às renegociações contratuais que podem ser dispendiosas e fazem com que as empresas não se desfaçam de seus ativos e, consequentemente, aumentem o grau de sticky costs.

Diferentemente dos quadros regionais anteriores, He, Teruya e Shimizu (2010) verificaram se as despesas com vendas, gerais e administrativas de empresas japonesas são assimétricas, quais os determinantes para tal assimetria, bem como o comportamento dos gestores após o colapso do mercado de ações em 1990. Encontraram que as despesas são sticky e que esse comportamento tende a aumentar com o crescimento econômico e com a intensidade dos ativos. Nessa mesma direção, com base no setor financeiro, Porporato e Werbin (2012) investigaram se os custos dos bancos da Argentina, Brasil e Canadá, durante o período de 2004 a 2009, são assimétricos. As análises indicam que bancos do Canadá, os quais possuem níveis maiores de intensidade de ativos, apresentam também maior assimetria.

Já, Dalla Via e Perego (2014) investigaram a assimetria dos custos laborais, do custo dos produtos vendidos e das despesas com vendas, gerais e administrativas em pequenas e médias empresas. Nas análises foram consideradas características das empresas, como a intensidade de ativos, além de outros aspectos, ao selecionar determinantes do comportamento assimétrico dos custos. Quanto a intensidade de ativos, os resultados são consistentes com os estudos anteriores para as despesas com vendas, gerais e administrativas das empresas de fabricação e para os custos laborais das empresas comerciais.

Ao analisar o comportamento dos custos antes e após a crise de 2008, Banker, Fang e Mehta (2013), também, forneceram algumas evidências sobre como a intensidade de ativos afeta o comportamento dos custos. De modo geral, para o período anterior à crise de 2008, verificaram que em empresas com alta intensidade de ativos fixos os custos apresentam, significativamente, maior grau de sticky costs do que em empresas com baixa intensidade de ativos fixos. Em 2009, período de crise econômica, ambas apresentaram comportamento antisticky, mas empresas com baixa intensidade de ativos fixos mostraram um grau significativamente maior de anti-sticky do que as empresas com alta intensidade de ativos fixos, o que evidencia a dificuldade de se desfazer desses ativos fixos, mesmo em períodos em que a redução de custos é almejada.

A relação entre incerteza sobre a demanda e o comportamento dos custos foi alvo de investigação de Banker, Byzalov e Plehn-Dujowich (2013). Num primeiro momento, as evidências encontradas suportam uma noção contraditória de que, sob maior incerteza em relação a demanda, as empresas do setor de fabricação procuraram manter níveis mais altos de capacidade fixa em termos de imóveis, instalações e equipamentos. Essa relação se justifica na medida em que as empresas buscam uma vantagem de mercado ao reduzir os custos 
relacionados ao ajuste dos recursos quando a incerteza apresenta períodos de aumento da demanda, ao manter estruturas de curto prazo mais rígidas.

Por meio de um modelo para cada hipótese relacionada a diferentes fatores, Jalilian e Elyssai (2014) analisaram os fatores que afetam o comportamento assimétrico das despesas com vendas, gerais e administrativas de empresas listadas na bolsa de Teerã. Com base nos resultados da intensidade de ativos, os autores argumentam que a assimetria dos custos é mais intensa em empresas maiores e com maior proporção de ativos fixos. Por fim, os mesmos autores argumentam que os gestores podem reduzir o grau de assimetria dos custos por meio de contratos de curto prazo de aluguéis de ativos operacionais, que permitem reduzir os custos de adaptação dos recursos em momentos de queda das vendas, devido a possibilidade de redução desses ativos de terceiros.

Em contexto semelhante, Abu-Serdaneh (2014) investigou se os custos dos produtos vendidos, bem como as despesas com vendas e administrativas de empresas listadas na bolsa de valores da Jordânia apresentam comportamento sticky ou anti-sticky. Os autores analisaram, também, o efeito de alguns possíveis fatores explicativos para o comportamento dos custos, como a intensidade de ativos, e identificaram que o custo dos produtos vendidos apresenta maior grau de assimetria para as empresas que possuem maior intensidade de ativos.

Paralelamente, no Brasil, alguns estudos analisaram a influência da intensidade de ativos no comportamento assimétrico dos custos como, por exemplo, Kremer (2015), que identificou a influência dos fatores explicativos para o comportamento assimétrico dos custos nas empresas brasileiras listadas na BM\&FBOVESPA inseridas em ambiente regulado, e considerou nas suas análises o grau de imobilização. No entanto, os resultados não foram conclusivos quanto a influência desse fator no comportamento do custo dos produtos vendidos e dos custos totais. Contudo, para as despesas, os resultados indicam que quanto maior o grau de imobilização, maior a assimetria no sentido sticky costs.

Já, Richartz (2016) verificou a influência de fatores explicativos no comportamento assimétrico dos custos das empresas listadas na BM\&FBOVESPA e incluiu em suas análises a intensidade de ativos, cujos resultados indicaram ser esse um dos fatores que maior influência no comportamento assimétrico dos custos e das despesas, no sentido sticky costs. Ainda, com base nas empresas listadas na BM\&FBOVESPA, ao determinar a interação conjunta entre os fatores explicativos da assimetria dos custos, Reis (2016) agregou os fatores explicativos em dois grupos de acordo com as suas interações, um que se refere ao aspecto inerente aos gestores e outro relacionado a estrutura empresarial, em que considerou a intensidade de ativos. E, Grejo (2016) analisou a influência do ativo imobilizado na assimetria dos custos de empresas listadas na BM\&FBOVESPA dos setores de consumo cíclico, materiais básicos e utilidades públicas, e concluiu que investimentos em ativo imobilizado podem gerar maior assimetria nos custos.

Por fim, com base nos estudos apresentados, é possível destacar a inclusão da intensidade de ativos em estudos nacionais e internacionais, os quais representam diferentes contextos para a explicação de um mesmo fenômeno. Além das pesquisas que inseriram a intensidade de ativos como um dos fatores principais em análise, ou como custos de ajustamento, alguns trabalhos incluíram tal variável como controle para encontrar outros determinantes para o comportamento dos custos e das despesas em empresas. Vale destacar que, em geral, tais estudos não verificaram a influência da intensidade de ativos na assimetria dos custos e, geralmente, não forneceram resultados relacionados a inclusão da variável.

No Quadro 1 apresenta-se um resumo dos trabalhos que inseriram a intensidade de ativos nas análises, segregados por função de utilização do fator, bem como a métrica (proxy) utilizada.

Conforme apresentado, o fator intensidade de ativos é utilizado com frequência, mas nem sempre como principal fator de análise. Geralmente, a variável aparece em estudos como uma variável de medida para custos de ajustamento ou como variável de controle. Ainda, a 
caracterização da variável como custos de ajustamento e como variável de controle podem estar relacionadas, uma vez que o uso na forma de variável de controle pode ter referência a custos de ajustamento.

Embora sejam utilizadas diferentes medidas nos estudos apresentados no Quadro 1, a maior parte dessas pesquisas encontrou coeficiente negativo e significativo para a intensidade de ativos, o que indica que quanto maior a intensidade de ativos maior a assimetria no sentido sticky costs. Com relação às medidas utilizadas para a concepção da intensidade de ativos, a proxy mais utilizada é a "razão do ativo total pela receita de vendas". No entanto, outras medidas são utilizadas para a verificação da intensidade de ativos, das quais as mais recorrentes são a "razão do imobilizado pela receita de vendas" e a "razão do imobilizado pelo ativo total".

Quadro 1 - Formas de emprego e medidas para intensidade de ativos

\begin{tabular}{|c|c|c|}
\hline Função & Autores & Proxy \\
\hline \multirow{14}{*}{$\begin{array}{c}\text { Intensidade } \\
\text { de ativos }\end{array}$} & Subramaniam e Weidenmier (2003) & $\begin{array}{l}\text { Ativos fixos/Vendas e Ativos fixos/Ativo } \\
\text { total }\end{array}$ \\
\hline & Calleja, Steliaros e Thomas (2006) & Ativo total \\
\hline & He, Teruya e Shimizu (2010) & Ativo/Vendas \\
\hline & Porporato e Werbin (2012) & Ativo total \\
\hline & Nassirzadeh et al. (2013) & Ativo total/Vendas líquidas \\
\hline & Banker, Fang e Mehta (2013) & Ativos fixos/Ativo total \\
\hline & Banker, Byzalov e Plehn-Dujowich (2013) & Ativos fixos \\
\hline & Dalla Via e Perego (2014) & Ativo total/Vendas \\
\hline & Abu-Serdaneh (2014) & Ativos/Vendas \\
\hline & Jalilian e Elyssai (2014) & Ativos fixos/Vendas \\
\hline & Kremer (2015) & Imobilizado/Ativo total \\
\hline & Richartz (2016) & Imobilizado/Ativo total \\
\hline & Reis (2016) & Imobilizado/Ativo total \\
\hline & Grejo (2016) & Imobilizado/Ativo total \\
\hline \multirow{6}{*}{$\begin{array}{l}\text { Custos de } \\
\text { ajustamento }\end{array}$} & Anderson, Banker e Janakiraman (2003) & Ativo total/Receita de vendas \\
\hline & Banker, Byzalovy e Plehn-Dujowichz (2010) & Ativos/Receita de vendas \\
\hline & Pichetkun (2012) & Ativo total/Vendas totais \\
\hline & Banker e Byzalov (2014) & Ativo total/Receita de vendas \\
\hline & Banker et al. (2014) & Ativo total/Vendas \\
\hline & Venieris, Naoum e Vlismas (2015) & Ativo total/Receita de vendas \\
\hline \multirow{13}{*}{$\begin{array}{c}\text { Variável de } \\
\text { controle }\end{array}$} & Banker, Ciftici e Mashruwala (2008) & Ativo total/Receita de vendas \\
\hline & Yasukata e Kajiwara (2011) & Giro do ativo imobilizado e Giro do estoque \\
\hline & Cheng, Jiang e Zeng (2011) & Ativos/Receita de vendas \\
\hline & Chen, Lu e Sougiannis (2012) & Ativo total/Receita de vendas \\
\hline & Banker, Byzalov e Chen (2013) & Ativo total/Vendas \\
\hline & Kama e Weiss (2013) & Ativo total/Receitas de vendas \\
\hline & Chen, Gores e Nasev (2013) & Ativo total/Vendas \\
\hline & Banker, Byzalov e Threinen (2013) & Ativo total/Vendas \\
\hline & Liang (2013) & Ativo total/Lucro operacional \\
\hline & Brüggen e Zehnder (2014) & Ativo total/Vendas \\
\hline & Holzhacker, Krishnan e Mahlendorf (2015) & Gastos com manutenção de ativos \\
\hline & Hall (2016) & Ativo imobilizado por dólar de receita \\
\hline & Banker et al. (2016) & Ativo total/Vendas \\
\hline
\end{tabular}

Fonte: elaborado pelos autores com base nos dados da pesquisa

Neste sentido, no presente trabalho, utilizam-se as medidas mais frequentemente destacadas na literatura, as quais são a razão do Ativo Total pela Receita Líquida de Vendas (AT/RLV), a razão do Imobilizado pela Receita Líquida de Vendas (I/RLV) e a razão do Imobilizado pelo Ativo Total (I/AT). 


\section{PROCEDIMENTOS METODOLÓGICOS}

O estudo foi realizado em empresas de capital aberto que negociaram suas ações na BM\&FBOVESPA, por um período de 21 anos, após a implantação do Plano Real, no Brasil. A seleção dessas empresas se justifica pela disponibilidade de informações necessárias para as análises, uma vez que são obrigadas à divulgação pública de relatórios econômico-financeiros. Vale destacar a exclusão do setor Finanças e Seguros, Fundos e Outros da amostra, conforme a classificação da base de dados utilizada, devido a estrutura operacional significativamente peculiar em relação aos demais setores abrangidos pela análise e regulamentação especial a que está submetido, fato que pode resultar em achados enviesados e impossibilitar a comparabilidade.

Os dados necessários para as análises foram coletados na base de dados Economática e dizem respeito a Receita Líquida de Vendas (RLV), ao Custo dos Produtos Vendidos (CPV), as Despesas Administrativas (DA), as Despesas com Vendas (DV), ao Ativo Total (AT), ao Imobilizado (I) e ao Setor de atuação. Para a análise do comportamento das despesas utilizouse o somatório das DV e DA, referidas nesse estudo como Despesas com Vendas e Administrativas (DVA).

Vale mencionar que a variação da RLV é utilizada como proxy para a variação do volume de atividades, prática comumente empregada em estudos dessa temática. Dalla Via e Perego (2014) advertem que os resultados gerados com base na aproximação do volume de atividades por meio da RLV devem ser interpretados com cautela devido às várias influências sobre a receita que não necessariamente estejam relacionadas às decisões dos gestores com relação ao volume de atividades, como variações nos preços e nos estoques mantidos pelas empresas. Para amenizar os efeitos de variações que não estejam relacionadas às atividades normais das empresas, excluiu-se das análises variações superiores a 50\% da RLV, do CPV e das DVA.

No sentido de verificar as medidas para a intensidade de ativos e suas respectivas influências no comportamento dos custos e das despesas, utilizou-se a análise de regressão para dados em painel, conforme o modelo de estimação dos sticky costs comumente empregado nesta temática e originário dos estudos de Anderson, Banker e Janakiraman (2003), no qual é possível verificar a variação dos custos e das despesas em função de $1 \%$ de variação na RLV. Com base nos critérios elucidados, as análises abrangem 454 empresas durante o período de 21 anos, as quais apresentaram ao menos dois anos de informações necessárias, devido a verificação da variação dos dados de um período em relação ao período passado. Como nem todas as empresas apresentaram informações para todos os anos, o painel é desbalanceado e resulta em 4.089 observações para as análises dos custos e 3.947 para as análises das despesas. Além da variação dos custos e das despesas, o modelo permite identificar se as variáveis inseridas são significativas e o quanto influenciam na assimetria.

Assim, para verificar quais das variáveis de intensidade dos ativos é mais aderente em termos de influência na assimetria dos gastos, para todos os tipos de empresas da amostra, utilizou-se o modelo de estimação dos sticky costs com as variáveis em teste como explicativas, sem considerar demais fatores. Logo, variáveis de controle não foram inseridas no modelo, o que se configura como uma limitação do estudo. Para adequação dos dados, realizou-se testes para estabelecer o modelo de estimação mais adequado, bem como analisou-se os pressupostos intrínsecos à regressão de dados em painel, e procedeu-se aos ajustes quando necessário.

Abaixo, apresentam-se os modelos para a análise da intensidade de ativos no Custos dos Produtos Vendidos:

1)

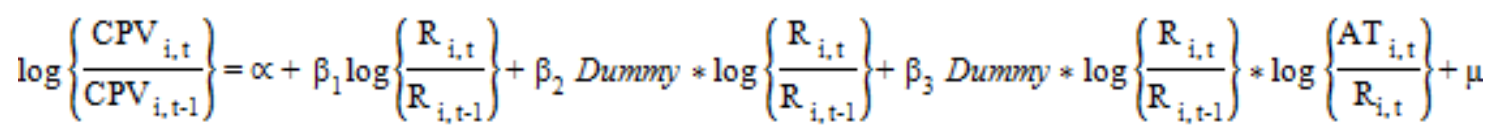


2)

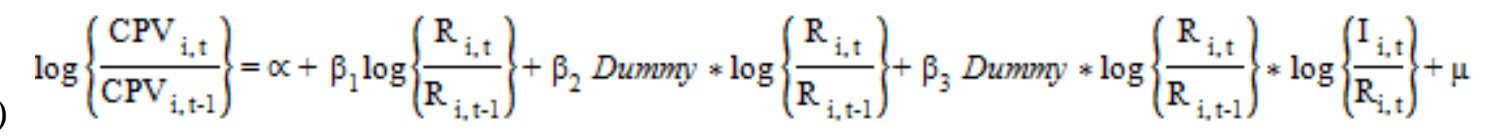

$$
\log \left\{\frac{\mathrm{CPV}_{\mathrm{i}, \mathrm{t}}}{\mathrm{CPV}_{\mathrm{i}, \mathrm{t}-1}}\right\}=\propto+\beta_{1} \log \left\{\frac{\mathrm{R}_{\mathrm{i}, \mathrm{t}}}{\mathrm{R}_{\mathrm{i}, \mathrm{t}-1}}\right\}+\beta_{2} \text { Dummy } * \log \left\{\frac{\mathrm{R}_{\mathrm{i}, \mathrm{t}}}{\mathrm{R}_{\mathrm{i}, \mathrm{t}-1}}\right\}+\beta_{3} \text { Dumwy } * \log \left\{\frac{\mathrm{R}_{\mathrm{i}, \mathrm{t}}}{\mathrm{R}_{\mathrm{i}, \mathrm{t}-1}}\right\} * \log \left\{\frac{\mathrm{I}_{\mathrm{i}, \mathrm{t}}}{\mathrm{AT}_{\mathrm{i}, \mathrm{t}}}\right\}+\mu
$$

Em seguida, destacam-se os modelos para a análise da intensidade de ativos nas Despesas com Vendas e Administrativas:

4)

$$
\begin{aligned}
& \log \left\{\frac{\mathrm{DVA}_{\mathrm{i}, \mathrm{t}}}{\mathrm{DVA}_{\mathrm{i}, \mathrm{t}-1}}\right\}=\propto+\beta_{1} \log \left\{\frac{\mathrm{R}_{\mathrm{i}, \mathrm{t}}}{\mathrm{R}_{\mathrm{i}, \mathrm{t}-1}}\right\}+\beta_{2} \text { Dummy } * \log \left\{\frac{\mathrm{R}_{\mathrm{i}, \mathrm{t}}}{\mathrm{R}_{\mathrm{i}, \mathrm{t}-1}}\right\}+\beta_{3} \text { Dummy } * \log \left\{\frac{\mathrm{R}_{\mathrm{i}, \mathrm{t}}}{\mathrm{R}_{\mathrm{i}, \mathrm{t}-1}}\right\} * \log \left\{\frac{\mathrm{AT}}{\mathrm{R}_{\mathrm{i}, \mathrm{t}}}\right\}+\mu \\
& \log \left\{\frac{\mathrm{DVA}_{\mathrm{i}, \mathrm{t}}}{\mathrm{DVA}_{\mathrm{i}, \mathrm{t}-1}}\right\}=\propto+\beta_{1} \log \left\{\frac{\mathrm{R}_{\mathrm{i}, \mathrm{t}}}{\mathrm{R}_{\mathrm{i}, \mathrm{t}-1}}\right\}+\beta_{2} \text { Dummy } * \log \left\{\frac{\mathrm{R}_{\mathrm{i}, \mathrm{t}}}{\mathrm{R}_{\mathrm{i}, \mathrm{t}-1}}\right\}+\beta_{3} D u m m y * \log \left\{\frac{\mathrm{R}_{\mathrm{i}, \mathrm{t}}}{\left.\mathrm{R}_{\mathrm{i}, \mathrm{t}-1}\right\} * \log \left\{\frac{\mathrm{I}_{\mathrm{i}, \mathrm{t}}}{\mathrm{R}_{\mathrm{i}, \mathrm{t}}}\right\}+\mu}\right. \\
& \log \left\{\frac{\mathrm{DVA}_{\mathrm{i}, \mathrm{t}}}{\mathrm{DVA}_{\mathrm{i}, \mathrm{t}-1}}\right\}=\propto+\beta_{1} \log \left\{\frac{\mathrm{R}_{\mathrm{i}, \mathrm{t}}}{\mathrm{R}_{\mathrm{i}, \mathrm{t}-1}}\right\}+\beta_{2} \text { Dummy } * \log \left\{\frac{\mathrm{R}_{\mathrm{i}, \mathrm{t}}}{\mathrm{R}_{\mathrm{i}, \mathrm{t}-1}}\right\}+\beta_{3} D u m m y * \log \left\{\frac{\mathrm{R}_{\mathrm{i}, \mathrm{t}}}{\left.\mathrm{R}_{\mathrm{i}, \mathrm{t}-1}\right\} * \log \left\{\frac{\mathrm{I}_{\mathrm{i}, \mathrm{t}}}{\mathrm{AT}_{\mathrm{i}, \mathrm{t}}}\right\}+\mu}\right.
\end{aligned}
$$

Por meio da constante $\alpha$, dos coeficientes angulares $\beta$, das variações da RLV - na equação representados por $\mathrm{R}$ - e do erro aleatório $\mu$ é possível verificar a variação dos custos e das despesas em decorrência das variações na receita. Além disso, o modelo evidencia o impacto dos fatores na variação dos custos, por meio dos coeficientes angulares, e se os mesmos são significativos para explicar as variações observadas, ao nível de 5\% de significância estabelecido.

Ainda, utilizou-se a aplicação do log das variações da R, do CPV, da DVA e das métricas para possibilitar a comparabilidade das variáveis inseridas entre as empresas analisadas e reduzir a heterocedasticidade dos dados, conforme Anderson, Banker e Janakiraman (2003). Vale destacar que a variável Dummy admite valor 1 quando da redução da RLV do período t em relação ao período t- 1 e valor 0 quando do aumento da RLV. Com base nos modelos apresentados, verifica-se qual a métrica mais representativa para a análise da intensidade de ativos, ao considerar e comparar a representatividade do coeficiente e da significância para cada uma delas.

\section{APRESENTAÇÃO E ANÁLISE DOS DADOS}

Com base nos procedimentos metodológicos adotados, apresentam-se as análises de cada proxy para a influência da intensidade de ativos no comportamento do CPV e das DVA. No entanto, para que os modelos de estimação de regressão com dados em painel apresentados nos procedimentos metodológicos, cujos resultados apresentam-se nas Tabelas de 1 a 6 , sejam considerados válidos, primeiramente foi necessário o atendimento aos pressupostos de regressão linear múltipla, por meio de testes de normalidade, multicolineariedade, heterocedasticidade e autocorrelação.

Em relação aos referidos testes, os resultados apresentaram curva de distribuição normal para os dados nos seis modelos desenvolvidos, o que permite identificar o pressuposto de normalidade. Para a multicolinearidade efetuou-se o teste VIF (Variance Inflation Factor), cujos resultados indicaram ausência do problema em todos os modelos analisados. Já, para a heterocedasticidade foi aplicado o teste de White, cujos resultados indicaram a sua presença nas 
variáveis independentes de todos os modelos e, portanto, procedeu-se a correção robusta quando da estimação dos modelos. O teste utilizado para a verificação da autocorrelação dos resíduos foi o de Wooldridge, que indicou a sua ausência em todos os modelos. Por fim, quanto a estimação dos modelos, realizou-se os testes de Chow, LM de Breusch e Pagan e o de Hausman para verificar qual é o modelo mais adequado para a estimação das regressões.

Assim, com base nos resultados dos testes aplicados, conclui-se que o modelo mais adequado para os custos é o de Efeitos Aleatórios, e o mais adequado para as despesas é o de Efeitos Fixos.

\subsection{Análise da intensidade de ativos no custo dos produtos vendidos}

A primeira proxy analisada trata da intensidade de ativos, obtida por meio da razão do Ativo Total pela Receita Líquida de Vendas, medida esta que foi utilizada primeiramente por Anderson, Banker e Janakiraman (2003) para as despesas, e comumente inserida em análises de assimetria dos custos e despesas por autores como os apresentados no Quadro 1. Desta forma, apresentam-se na Tabela 1, os resultados das análises da proxy razão do Ativo Total pela Receita Líquida de Vendas (AT/RLV).

Tabela 1 - Influência da proxy AT/RLV

\begin{tabular}{|c|c|c|c|c|}
\hline lvcpv & coef. & std. err. & $\mathbf{Z}$ & $\mathbf{p}>\mathbf{z}$ \\
\hline lvrlv & 0,7716 & 0,02 & 33,18 & 0,00 \\
\hline dlvrlv & 0,1197 & 0,04 & 2,82 & 0,01 \\
\hline at_rlv & $-0,1743$ & 0,05 & $-3,50$ & 0,00 \\
\hline cons & $-0,0011$ & 0,00 & $-0,80$ & 0,43 \\
\hline $\mathrm{r}^{2}$ within & 0,6019 & & Wald chi2 & 3810,18 \\
\hline $\mathrm{r}^{2}$ between & 0,5923 & & Prob $>$ chi 2 & 0,0000 \\
\hline $\mathrm{r}^{2}$ overall & 0,6239 & & & \\
\hline
\end{tabular}

Nota:

lvcpv $=\log$ da variação dos custos dos produtos vendidos (CPV)

lvrlv = log da variação da receita líquida de vendas (RLV)

dlvrlv = dummy de redução do log da variação da RLV

at_rlv = ativo total/receita líquida de vendas

Foram feitos ajustes nas situações em que os dados não atendiam aos pressupostos do modelo de regressão.

Fonte: elaborado pelos autores com base nos dados da pesquisa

Tabela 2 - Influência da proxy I/RLV

\begin{tabular}{c|cccc}
\hline lvcpv & coef. & std. err. & $\mathbf{z}>\mathbf{z}$ & 0,00 \\
\hline lvrlv & 0,7769 & 0,02 & 33,36 \\
dlvrlv & $-0,0092$ & 0,04 & $-0,23$ & 0,82 \\
i_rlv & $-0,0951$ & 0,04 & $-2,64$ & 0,01 \\
cons & $-0,0012$ & 0,00 & $-0,85$ & 0,40 \\
\hline r $^{2}$ within & 0,5985 & & Wald chi2 & 0,0000 \\
r $^{2}$ between & 0,5835 & & Prob chi2 \\
\hline$r^{2}$ overall & 0,6221 & &
\end{tabular}

Nota:

lvcpv = log da variação dos custos dos produtos vendidos (CPV)

lvrlv = log da variação da receita líquida de vendas (RLV)

dlvrlv = dummy de redução do $\log$ da variação da RLV

at_rlv = imobilizado/receita líquida de vendas

Foram feitos ajustes nas situações em que os dados não atendiam aos pressupostos do modelo de regressão.

Fonte: elaborado pelos autores com base nos dados da pesquisa

Ao considerar o coeficiente negativo de -0,1743 e a significância de 0,00 , quanto maior a proporção de Ativo Total em relação a Receita Líquida de Vendas, maior a assimetria do CPV no sentido sticky costs. Esses resultados corroboram os achados de Banker, Byzalov e PlehnDujowichz (2010), Kama e Weiss (2012), Banker et al. (2012), Banker, Byzalov e Threinen 
(2013), que encontraram coeficientes significativos, e negativos, para a intensidade de ativos por meio desta proxy na análise do comportamento dos custos.

Os resultados da métrica razão do Imobilizado pela Receita Líquida de Vendas (I/RLV) encontram-se na Tabela 2.

Similarmente a proxy AT/RLV, o I/RLV apresenta coeficiente -0,0951 e significância de 0,01 , o que permite concluir que quanto maior a proporção do Imobilizado em relação a RLV, maior a assimetria do CPV no sentido sticky costs, como encontrado por Subramaniam e Weidenmier (2003) para custos operacionais. Assim, esta também é uma medida que influencia a assimetria no CPV, embora com menor impacto quando comparada a métrica anterior.

Por fim, na Tabela 3, apresentam-se os resultados da proxy razão do Imobilizado pelo Ativo Total (I/AT).

Tabela 3 - Influência da proxy I/AT

\begin{tabular}{c|cccc}
\hline lvcpv & coef. & std. err. & $\mathbf{z}$ & $\mathbf{p > \mathbf { Z }}$ \\
\hline lvrlv & 0,7804 & 0,02 & 33,36 & 0,00 \\
dlvrlv & 0,0306 & 0,04 & 0,69 & 0,49 \\
i_at & 0,0185 & 0,03 & 0,56 & 0,58 \\
cons & $-0,0015$ & 0,00 & $-1,02$ & 0,31 \\
\hline $\mathrm{r}^{2}$ within & 0,5958 & & Wald chi2 & 2953,15 \\
$\mathrm{r}^{2}$ between & 0,5813 & & Prob>chi2 & 0,0000 \\
$\mathrm{r}^{2}$ overall & 0,6203 & & & \\
\hline
\end{tabular}

Nota:

lvcpv $=\log$ da variação dos custos dos produtos vendidos $(\mathrm{CPV})$

lvrlv $=\log$ da variação da receita líquida de vendas (RLV)

dlvrlv = dummy de redução do log da variação da RLV

i_at = imobilizado/ativo total

Foram feitos ajustes nas situações em que os dados não atendiam aos pressupostos do modelo de regressão.

Fonte: elaborado pelos autores com base nos dados da pesquisa

Com relação a proxy I/AT, a significância de 0,58 não permite afirmar que seja uma métrica de influência na assimetria do CPV nas empresas analisadas, uma vez que a proxy não é significativa ao nível de $95 \%$ de confiança estabelecido. No entanto, nas análises de Subramaniam e Weidenmier (2003) e de Richartz (2016) a variável intensidade de ativos, medida por meio do Imobilizado divido pelo Ativo Total, apresentou-se negativa e significativa.

Assim, com base nos resultados das três métricas apresentadas, é possível verificar que a proxy mais significativa e com o maior coeficiente é a razão do Ativo Total pela Receita Líquida de Vendas (AT/RLV). Essa métrica, além de ser a de maior influência na assimetria do Custo dos Produtos Vendidos, de modo geral, é a mais comumente utilizada pelos autores quando da verificação da influência da intensidade de ativos na assimetria dos custos e das despesas.

\subsection{Análise da intensidade de ativos nas despesas com vendas e administrativas}

A intensidade de ativos obtida por meio da razão do Ativo Total pela Receita Líquida de Vendas é, também, frequentemente utilizada para análise das despesas, como no estudo seminal de Anderson, Banker e Janakiraman (2003) e, posteriormente, por Banker, Ciftici e Mashruwala (2008), Banker, Byzalovy e Plehn-Dujowichz (2010), He, Teruya e Shimizu (2010), Cheng, Jiang e Zeng (2011), Banker et al. (2012), Chen, Lu e Sougiannis (2012), Pichetkun (2012), Banker, Byzalov e Threinen (2013), Chen, Gores e Nasev (2013), Kama e Weiss (2013), Nassirzadeh et al. (2013), Abu-Serdaneh (2014), Banker e Byzalov (2014), 
Banker et al. (2014), Brüggen e Zehnder (2014), Dalla Via e Perego (2014), Venieris, Naoum e Vlismas (2015) e Banker et al. (2016).

Os resultados relacionados à medida razão do Ativo Total pela Receita Líquida de Vendas (AT/RLV) são elucidados na Tabela 4.

O coeficiente de -0,1546 com significância de 0,01 para as DVA indica que, assim como para o CPV, a proporção do Ativo Total em relação a Receita Líquida de Vendas está positivamente associada aos sticky costs. Estes achados corroboram os resultados de Anderson, Banker e Janakiraman (2003), Banker, Ciftici e Mashruwala (2008) e Chen, Lu e Sougiannis (2012), que identificaram coeficientes negativos, e significativos, para esta medida quando da análise das despesas.

Tabela 4 - Influência da proxy AT/RLV

\begin{tabular}{c|cccc}
\hline lvdva & coef. & std. err. & t & p>t \\
\hline lvrlv & 0,3239 & 0,04 & 7,70 & 0,00 \\
dlvrlv & 0,1787 & 0,07 & 2,57 & 0,01 \\
at_rlv & $-0,1546$ & 0,06 & $-2,45$ & 0,01 \\
cons & $-0,0048$ & 0,00 & $-2,48$ & 0,01 \\
\hline r $^{2}$ within & 0,1324 & & F & 115,66 \\
r $^{2}$ between & 0,1377 & & Prob $>$ F & 0,0000 \\
r $^{2}$ overall & 0,1522 & & & \\
\hline
\end{tabular}

Nota:

lvdva = log da variação das despesas com vendas e administrativas (DVA)

lvrlv = log da variação da receita líquida de vendas (RLV)

dlvrlv = dummy de redução do log da variação da RLV

at_rlv = ativo total/receita líquida de vendas

Foram feitos ajustes nas situações em que os dados não atendiam aos pressupostos do modelo de regressão.

Fonte: elaborado pelos autores com base nos dados da pesquisa

Já, para a análise da influência da intensidade de ativos por meio da razão do Imobilizado pela Receita Líquida de Vendas (I/RLV) apresenta-se a Tabela 5.

Tabela 5 - Influência da proxy I/RLV

\begin{tabular}{c|cccc}
\hline Lvdva & coef. & std. err. & t & p>t \\
\hline Lvrlv & 0,3307 & 0,04 & 7,82 & 0,00 \\
Dlvrlv & 0,0876 & 0,07 & 1,24 & 0,21 \\
i_rlv & 0,0130 & 0,05 & 0,27 & 0,79 \\
Cons & $-0,0052$ & 0,00 & $-2,63$ & 0,01 \\
\hline $\mathrm{r}^{2}$ within & 0,1274 & & F & 103,32 \\
r $^{2}$ between & 0,1436 & & & 0,0000 \\
r $^{2}$ overall & 0,1515 & & & \\
\hline
\end{tabular}

Nota:

lvdva = log da variação das despesas com vendas e administrativas (DVA)

lvrlv = log da variação da receita líquida de vendas (RLV)

dlvrlv = dummy de redução do log da variação da RLV

i_rlv = imobilizado/receita líquida de vendas

Foram feitos ajustes nas situações em que os dados não atendiam aos pressupostos do modelo de regressão.

Fonte: elaborado pelos autores com base nos dados da pesquisa

Para a proxy I/RLV o coeficiente de 0,0130 não se mostra significativo devido a significância de 0,79, o que indica que esta métrica não influencia na assimetria das Despesas com Vendas e Administrativas das empresas analisadas, embora seja significativa em outros estudos, como Subramaniam e Weidenmier (2003).

Por fim, com relação a medida da razão do Imobilizado pelo Ativo Total (I/AT), apresentam-se os resultados na Tabela 6.

Para a proxy I/AT o coeficiente é significativo, no entanto, é positivo. Estes resultados indicam que a intensidade de ativos verificada por meio da razão do Imobilizado pelo Ativo 
Total influencia no comportamento assimétrico das despesas no sentido anti-sticky costs. Assim, os resultados corroboram aos achados de Subramaniam e Weidenmier (2003) e Richartz (2016).

Da mesma forma que na análise do CPV, os resultados apresentados indicam que a proxy de maior influência no comportamento assimétrico das DVA é a razão do Ativo Total pela Receita Líquida de Vendas. O Quadro 2 sintetiza os principais achados da pesquisa, ao indicar o coeficiente e a respectiva significância de cada uma das medidas analisadas para a intensidade de ativos no CPV e nas DVA.

Tabela 6 - Influência da proxy I/AT

\begin{tabular}{c|cccc}
\hline Lvdva & coef. & std. err. & t & p>t \\
\hline lvrlv & 0,3310 & 0,04 & 7,83 & 0,00 \\
dlvrlv & 0,1893 & 0,07 & 2,70 & 0,01 \\
i_at & 0,1235 & 0,04 & 3,24 & 0,00 \\
cons & $-0,0051$ & 0,00 & $-2,64$ & 0,01 \\
\hline $\mathrm{r}^{2}$ within & 0,1315 & & $\mathrm{~F}$ & 111,84 \\
$\mathrm{r}^{2}$ between & 0,1251 & & Prob $>$ F & 0,0000 \\
r $^{2}$ overall & 0,1536 & & & \\
\hline
\end{tabular}

\section{Nota:}

lvdva = log da variação das despesas com vendas e administrativas (DVA)

lvrlv = log da variação da receita líquida de vendas (RLV)

dlvrlv = dummy de redução do log da variação da RLV

i_at = imobilizado/ativo total

Foram feitos ajustes nas situações em que os dados não atendiam aos pressupostos do modelo de regressão.

Fonte: elaborado pelos autores com base nos dados da pesquisa

Quadro 2 - Resumo dos resultados

\begin{tabular}{|c|c|c|c|}
\hline Proxy & Resultado & CPV & DVA \\
\hline \multirow{3}{*}{ AT/RLV } & Direção da assimetria & Sticky Costs & Sticky Costs \\
& Coeficiente & $-0,1743$ & $-0,1546$ \\
& $\mathrm{p}>\mathrm{t}$ & 0,00 & 0,01 \\
\hline \multirow{3}{*}{ I/RLV } & Direção da assimetria & Sticky Costs & Não significante \\
& Coeficiente & $-0,0951$ & 0,0130 \\
& $\mathrm{p}>\mathrm{t}$ & 0,01 & 0,79 \\
\hline \multirow{3}{*}{ I/AT } & Direção da assimetria & Não significante & Anti-sticky Costs \\
& Coeficiente & 0,0185 & 0,1235 \\
& $\mathrm{p}>\mathrm{t}$ & 0,58 & 0,00 \\
\hline
\end{tabular}

Nota:

at/rlv = ativo total/receita líquida de vendas

$\mathrm{i} / \mathrm{rlv}=$ imobilizado/receita líquida de vendas

i_at $=$ imobilizado/ativo total

dva $=$ despesas com vendas e administrativas

$\mathrm{cpv}=$ custo dos produtos vendidos

Fonte: elaborado pelos autores com base nos dados da pesquisa

Conforme já destacado, e observado no Quadro 2, a intensidade de ativos exerce influência na assimetria dos custos e das despesas, quando verificada pela proxy AT/RLV. No entanto, a métrica I/RLV para o comportamento dos custos e I/AT para o comportamento das despesas, também, exercem influência na assimetria, porém com menor intensidade quando comparadas com a proxy AT/RLV. As demais medidas, I/AT para os custos e I/RLV para as despesas não se apresentaram estatisticamente significantes para as análises realizadas.

Destaca-se que o uso irrestrito de diferentes métricas como proxy para um mesmo conceito de intensidade de ativos utilizado na literatura de comportamento dos custos pode trazer importantes distorções nas discussões dos achados. Assim, os resultados do presente estudo evidenciam que, para o caso do Brasil, a métrica que apresenta maior aderência em termos de influência no comportamento sticky cost é a razão entre Ativo Total e Receita Líquida 
de Vendas (AT/RLV), a qual pode ser interpretada como o valor total dos ativos das empresas ponderado pelo seu tamanho em termos de faturamento. Diversos estudos internacionais fizeram o uso dessa métrica para evidenciar o impacto da intensidade de ativos no comportamento cost stickyness como He, Teruya e Shimizu (2010), Abu-Serdaneh (2014) e Anderson, Banker e Janakiraman (2003).

A utilização das outras métricas analisadas nesta pesquisa como fator explicativo para o comportamento dos custos, também, tem seu valor informacional. A razão do imobilizado pela receita pode trazer uma ideia de dimensionamento do ativo fixo ponderado pelo tamanho da organização em termos de faturamento, enquanto a razão do imobilizado pelo ativo total oferece a concepção da parcela do ativo que é fixa e sobre a qual se tem maiores custos de ajustamento para modificá-la. Ao mesmo tempo, todas essas variáveis trazem a concepção de que quanto mais ativos a empresa possui, maiores são seus custos de ajustamento. E, por consequência, maior a possibilidade de a empresa apresentar assimetria no comportamento dos custos.

\section{CONCLUSÃO}

Os resultados sobre o comportamento assimétrico dos custos têm implicações para os decisores corporativos, na medida em que decisões com base em modelos tradicionais superestimam ou subestimam a capacidade de resposta dos custos frente às alterações nos níveis de atividade, conforme ressaltam Calleja, Steliaros e Thomas (2006). Nesta direção, o presente estudo contribui para o entendimento do comportamento assimétrico dos custos e das despesas por meio da análise de um dos fatores explicativos mais importantes ressaltados na literatura, sobretudo com relação a influência da intensidade de ativos e as métricas utilizadas para a sua compreensão, com base nas proxies mais recorrentes e aplicados em diferentes contextos regionais.

Assim, para o Custo dos Produtos Vendidos, os resultados do presente estudo indicam que a razão do Ativo Total pela Receita Líquida de Vendas é a proxy, para a intensidade de ativos, de maior influência sobre o comportamento assimétrico, na medida em que se apresentou significativa e com o maior coeficiente quando comparada com as demais. A proxy razão do Imobilizado pela Receita Líquida de Vendas, também, apresentou coeficiente significativo, porém menor em relação ao coeficiente da proxy anterior. Por outro lado, a razão do Imobilizado pelo Ativo Total não se apresentou significativa, o que não permite afirmar que esta seja uma proxy que influencia na assimetria dos custos das empresas analisadas.

Quanto às Despesas com Vendas e Administrativas, a métrica para a intensidade de ativos obtida por meio da razão do Ativo Total pela Receita Líquida de Vendas é, também, a mais representativa, ao exercer maior influência no comportamento assimétrico dos custos, devido a sua significância e por ter o maior coeficiente. Já, a razão do Imobilizado pela Receita Líquida de Vendas não se apresentou significativa na análise das despesas, enquanto que a razão do Imobilizado pelo Ativo Total mostrou-se significativo, mas com influência no sentido anti-sticky costs e coeficiente menor em relação à proxy Ativo Total pela Receita Líquida de Vendas.

Em conclusão, na confrontação dos resultados dos testes realizados como possibilidades de métricas para a intensidade de ativos, sobretudo, derivados dos conceitos da literatura e aplicados em diferentes contextos, regionais e organizacionais, é possível verificar que medidas e conceitos não são necessariamente absolutos em termos de definição, mas existem alguns mais adequados e que se tornam usuais devido a sua representatividade. No entanto, diferentes conceitos e medidas podem ser melhores aplicados a diferentes ambientes e, também, podem ser ajustados caso se tornem deficientes. Assim, os achados do presente estudo fornecem uma contribuição para a linha de pesquisa em comportamento dos custos por discutir os benefícios informacionais no uso das diferentes métricas para a intensidade de ativos e inferir sobre qual 
delas se mostra mais representativa, o que pode servir de suporte para as pesquisas nacionais e internacionais nas quais uma proxy para intensidade de ativos é aplicada.

Uma compreensão do comportamento assimétrico dos custos resulta num robusto sistema de planejamento e controle, o qual pode atenuar o efeito dos sticky costs. Para evitar ou minimizar tal efeito, os gerentes podem identificar e gerenciar a capacidade e os recursos não utilizados, não necessariamente por meio da redução dos mesmos, mas por formas alternativas como a transferência de recursos não utilizados para outras atividades (CALLEJA; STELIAROS; THOMAS, 2006).

Por fim, conforme verificado na literatura, a intensidade de ativos é comumente utilizada para medir os custos de ajustamento, por meio da sua junção com outros fatores, como a intensidade de empregados. No entanto, a combinação dos fatores utilizados para a verificação dos custos de ajustamento nem sempre é padronizada. Assim, o estudo das variáveis utilizadas para os custos de ajustamento e as combinações mais recorrentes são sugestões para futuros trabalhos.

\section{REFERÊNCIAS}

ABU-SERDANEH, J. The asymmetrical behavior of cost: evidence from Jordan.

International Business Research, v. 7, n. 8, p. 113, 2014.

ANDERSON, M. C.; BANKER, R. D.; JANAKIRAMAN, S. N. Are selling, general, and administrative costs "sticky"? Journal of Accounting Research, v. 41, n. 1, p. 47-63, 2003.

BANKER, R. D.; BASU, S.; BYZALOV, D.; CHEN, J. Y. The confounding effect of cost stickiness on conservatism estimates. Journal of Accounting and Economics, v. 61, n. 1, p. 203-220, 2016.

BANKER, R. D.; BYZALOV, D. Asymmetric cost behavior. Journal of Management Accounting Research, v. 26, n. 2, p. 43-79, 2014.

BANKER, R. D.; BYZALOV, D.; CIFTCI, M.; MASHRUWALA, R. The moderating effect of prior sales changes on asymmetric cost behavior. Journal of Management Accounting Research, v. 26, n. 2, p. 221-242, 2014.

BANKER, R. D.; BYZALOV, D.; PLEHN-DUJOWICH, J. M. Sticky cost behavior: theory and evidence. In: AAA 2011 Management Accounting Section (MAS), Meeting Paper, 2010.

BANKER, R. D.; BYZALOV, D.; PLEHN-DUJOWICH, J. M. Demand uncertainty and cost behavior. The Accounting Review, v. 89, n. 3, p. 839-865, 2013.

BANKER, R. D.; BYZALOV, D.; THREINEN, L. Determinants of international differences in asymmetric cost behavior. Fox School of Business, v. 1, 2013.

BANKER, R. D.; CIFTCI, M.; MASHRUWALA, R. Managerial optimism, prior period sales changes, and sticky cost behavior. Working Paper, 2008. Disponível em: <http://ssrn.com.ololo.sci-hub.cc/abstract=1599284>. Acesso em: 07/04/2016.

BANKER, R. D.; FANG, S.; MEHTA, M. Cost behavior during the world economic crisis. Working paper, Temple University, 2013.

BRÜGGEN, A.; ZEHNDER, J. O. SG\&A cost stickiness and equity-based executive compensation: does empire building matter? Journal of Management Control, v. 25, n. 3-4, 
p. 169-192, 2014.

CALLEJA, K.; STELIAROS, M.; THOMAS, D. C. A note on cost stickiness: some international comparisons. Management Accounting Research, v. 17, n. 2, p. 127-140, 2006.

CANNING, J. B. The economics of accountancy: a critical analysis of accounting theory. The Ronald Press Company, 1929.

CHEN, C. X.; GORES, T.; NASEV, J. Managerial overconfidence and cost stickiness. Science Research Network, 2013. Disponível em: < http://ssrn.com/abstract=2208622>. Acesso em: 10 maio 2016.

CHENG, S.; JIANG, W.; ZENG, Y. Cost stickiness in large versus small firms. Social Science Research Network, 2011. Disponível em: < http://ssrn.com/abstract=1910039>. Acesso em: 10 maio 2016.

CHEN, C. X.; LU, H.; SOUGIANNIS, T. The agency problem, corporate governance, and the asymmetrical behavior of selling, general, and administrative costs. Contemporary

Accounting Research, v. 29, n. 1, p. 252-282, 2012.

COMITÊ DE PRONUNCIAMENTOS CONTÁBEIS. Pronunciamento Conceitual Básico CPC 00 (R1). Estrutura Conceitual para Elaboração e Divulgação de Relatório ContábilFinanceiro. 2011.

DALLA VIA, N.; PEREGO, P. Sticky cost behaviour: evidence from small and medium sized companies. Accounting \& Finance, v. 54, n. 3, p. 753-778, 2014.

GREJO, L. M. A influência do ativo imobilizado no comportamento assimétrico dos custos. 2016. 74 f. Dissertação (Mestrado em Ciências Contábeis) - Curso de Pós-Graduação em Ciências Contábeis, Universidade Estadual de Maringá, Maringá, Brasil.

HALL, C. M. Does ownership structure affect labor decisions? The Accounting Review, v. 91, n. 6, p. 1671-1696, 2016.

HE, D.; TERUYA, J.; SHIMIZU, T. Sticky selling, general and administrative cost behavior and its changes in Japan. Global Journal of Business Research, v. 4, n. 4, p. 1-10, 2010

HENDRIKSEN, E. S.; VAN BREDA, M. F. Teoria da contabilidade. São Paulo: Atlas, 2009.

HOLZHACKER, M.; KRISHNAN, R.; MAHLENDORF, M. D. The impact of changes in regulation on cost behavior. Contemporary Accounting Research, v. 32, n. 2, p. 534-566, 2015.

JALILIAN, M.; ELYSSAI, E. V. Review and analyzing the factors affecting the stickiness behavior of general administration costs and sales of listed companies in Tehran Stock Exchange. Applied mathematics in Engineering, Management and Technology, v. 2, n. 4, p. 584-596, 2014.

KAMA, I.; WEISS, D. Do earnings targets and managerial incentives affect sticky costs? Journal of Accounting Research, v. 51, n. 1, p. 201-224, 2013. 
KREMER, A. W. Análise de fatores explicativos para o comportamento assimétrico dos custos em ambiente regulado. 2015. 81 f. Dissertação (Mestrado em Contabilidade) - Curso de Pós-Graduação em Contabilidade, Universidade Federal de Santa Catarina, Florianópolis, Brasil.

LI, L. Study of corporate governance on relation between self-interest incentive and cost stickiness. International Journal of Digital Content Technology and its Applications, v. 7, n. 6, p. 706, 2013.

NASSIRZADEH, F.; SAEI, M. J.; SALEHI, M.; HADDAD, A. A Study of the stickiness of cost of goods sold and operating costs to changes in sales level in Iran. Studies in Business and Economics, v. 8, n. 2, p. 79-89, 2013.

PICHETKUN, N. The determinants of sticky cost behavior on political costs, agency costs, and corporate governance perspectives. 2012. Tese (Doutorado em Administração) Faculty of Business Administration, Rajamangala University of Technology Thanyaburi. Disponível em: <http://www.repository.rmutt.ac.th/handle/123456789/1245>. Acesso em: 20 maio 2016.

PORPORATO, M.; WERBIN, E. M. Active cost management in banks: evidence of sticky costs in Argentina, Brazil and Canada. International Journal of Financial Services Management, v. 5, n. 4, p. 303-320, 2012.

REIS, L. S. Comportamento assimétrico dos custos análise conjunta dos fatores explicativos. 2016. 91f. Dissertação (Mestrado em Contabilidade) - Curso de Pós-Graduação em Contabilidade, Universidade Federal de Santa Catarina, Florianópolis, Brasil.

RICHARTZ, F. Fatores explicativos para o comportamento assimétrico dos custos das empresas brasileiras. 2016. 157f. Tese (Doutorado em Contabilidade) - Curso de PósGraduação em Contabilidade, Universidade Federal de Santa Catarina, Florianópolis, Brasil.

SILVA, A. DA; ZONATTO, V. C. DA S.; MAGRO, C. B. D.; KLANN, R. Sticky costs behavior and earnings management. Brazilian Business Review, v. 16, n. 2, p. 191-206, 2019.

SUBRAMANIAM, C.; WEIDENMIER, M. L. Additional evidence on the sticky behavior of costs. Social Science Research Network, 2003. Disponível em: <https://ssrn.com/abstract=369941>. Acesso em: 10 maio 2016.

VENIERIS, G.; NAOUM, V. C.; VLISMAS, O. Organisation capital and sticky behaviour of selling, general and administrative expenses. Management Accounting Research, v. 26, p. 54-82, 2015.

WEISS, D. Cost behavior and analysts' earnings forecasts. The Accounting Review, v. 85, n. 4, p. 1441-1471, 2010.

YASUKATA, K.; KAJIWARA, T. Are 'Sticky Costs' the Result of Deliberate Decision of Managers? Social Science Research Network, 2011. Disponível em:

$<$ https://ssrn.com/abstract=1444746>. Acesso em: 10 maio 2016. 